\title{
Ethnologies
}

Gradhiva. Revue semestrielle d'anthropologie et de muséologie, numéro 1, nouvelle série. Dossier Haïti et l'anthropologie. (Paris, Musée du Quai Branly, mai 2005. Pp. 272, ISBN 2-915133-08-5)

\section{Marie Meudec}

Volume 28, numéro 1, 2006

Haïti - Face au passé

Haïti - Confronting the Past

URI : https://id.erudit.org/iderudit/014160ar

DOI : https://doi.org/10.7202/014160ar

Aller au sommaire du numéro

Éditeur(s)

Association Canadienne d'Ethnologie et de Folklore

ISSN

1481-5974 (imprimé)

1708-0401 (numérique)

Découvrir la revue

Citer ce compte rendu

Meudec, M. (2006). Compte rendu de [Gradhiva. Revue semestrielle

d'anthropologie et de muséologie, numéro 1, nouvelle série. Dossier Haïti et

l'anthropologie. (Paris, Musée du Quai Branly, mai 2005. Pp. 272, ISBN

2-915133-08-5)]. Ethnologies, 28(1), 285-288. https://doi.org/10.7202/014160ar d'utilisation que vous pouvez consulter en ligne.

https://apropos.erudit.org/fr/usagers/politique-dutilisation/ 
chaque nuit, depuis le gouffre de ses cauchemars... Enfin, de petite touche en petite touche, suivant une technique narrative presque pointilliste, le portrait du bourreau se précise. Le puzzle se reconstitue. Progressant de façon concentrique, le roman atteint sa cible : les affres du remords et de la culpabilité endurées par un ex-milicien que torture sa mémoire clandestine. Alors, et alors seulement, au fil du dernier chapitre, l'origine de la cicatrice qui zèbre son visage nous est révélée et, avec elle, les détails de son dernier crime.

Au terme de la lecture, on peut affirmer qu'Edwidge Danticat réussit à dresser un portrait saisissant des résonances multiples que peuvent engendrer dans la vie quotidienne de tout un peuple un régime de terreur, qui laisse trop souvent le meurtre impuni. Peut-être parce qu'il s'ancre sur "l'autre rive" (états-unienne), le regard que pose la romancière sur les dérives totalitaires haïtiennes se veut non pas réquisitoire, mais plaidoyer pour une humanité faillible. Profondément humaniste, Edwidge Danticat n'accable pas le bourreau, qui revêt ici la figure d'un père. Sans banaliser l'horreur des gestes qui furent posés et qu'elle persiste à considérer comme impardonnables —, la romancière nous invite à imaginer, avec elle, la silhouette d'un tortionnaire sauvé par l'amour d'une femme et d'un enfant.

Katell Colin-Thebaudeau

Université Laval

Québec

\section{Gradhiva. Revue semestrielle d'anthropologie et de muséologie, numéro 1, nouvelle série. Dossier Haiiti et l'anthropologie. (Paris, Musée du Quai Branly, mai 2005. Pp. 272, ISBN 2-915133-08-5)}

Belle transition pour cette nouvelle série de la revue, publiée précédemment par Jean-Michel Place et éditée dorénavant par le Musée du Quai Branly, qui conserve toutefois une structure identique regroupant des essais, études originales, archives — inédites pour certaines - et témoignages d'anthropologues, d'historiens, d'épistémologues, d'historiens de l'art ou de la littérature. Ce double numéro, consacré à l'histoire et aux développements actuels de l'anthropologie en Haïti, débute par trois textes qui s'inscrivent dans une perspective comparative. Le premier est une discussion disciplinaire, au sujet de «l'entre-deux » reliant l'anthropologie et l'histoire, de Marcel 
Detienne, qui avance des propositions de réponse à la question «Comment comparer l'incomparable? », abordant en ces termes la quête d'un « comparatisme expérimental et constructif » du politique ; comparatisme que le deuxième pourrait qualifier de "géographique ", entre Haïti et la France, et ce à partir d'une conférence donnée par le poète franco-haïtien René Depestre au sujet de son parcours et de son analyse de la société haïtienne; le troisième est un témoignage de ce même écrivain recueilli par Jean-Luc Bonniol sur les figures de proue en Haïti et le poids de l'anthropologie dans la construction nationale.

Adoptant une perspective plus historique et localement située, le dossier établi par Carlo Avierl Célius contient de nombreuses études portant sur la constitution de l'anthropologie en Haïti, nécessairement liée à la question raciale et à la situation politique et religieuse. Que ce soit à partir de récits d'anthropologues locaux tels qu'Anténor Firmin, Jean Price-Mars, Jacques Roumain, Suzanne Comhaire-Sylvain, Jean Chrisostome Dorsainvil, Rémy Bastien, ou étrangers comme Roger Bastide, Michel Leiris, Melville Herskovits, Alfred Métraux, ou d'une institution (Bureau d'ethnologie de Port-au-Prince), le dossier donne la place à une série d'articles questionnant les modes de représentation liés à la création artistique, au vodou - en tant que phénomène religieux, politique et artistique —, ainsi qu'à la culture et à la société haïtienne comme lieux d'investigation anthropologique.

Paradigme au fondement de l'anthropologie, la problématique de la race est présente en Haïti dès les premiers temps de la construction coloniale du Nouveau Monde. Adoptant cette perspective, Carlo A. Célius montre ainsi que la discipline anthropologique peut être considérée comme «consubstantielle à l'histoire d'Haïti ». Dès lors, les concepts fondateurs de l'ethnologie doivent être explicités si l'on veut comprendre en quoi cette vision du monde constitue « la formation discursive dominante du XX ${ }^{\mathrm{e}}$ siècle haïtien ». Quant aux liens entre création artistique et espace discursif des ethnologues en Haïti, ces domaines nécessitent tous deux une recontextualisation au sein du paradigme évolutionniste de départ, lequel s'est déplacé progressivement, au sein de l'avènement d'un "art naïf », d'une conception civilisationnelle de l'altérité au primitivisme, tel que conçu par l'anthropologie puis élargi à l'univers esthétique. Par la suite, Rachel Beauvoir-Dominique nous fait découvrir la collection privée rassemblée depuis 1980 par Marianne Lehmann, analysant sa portée indubitablement ethnologique et esthétique, et retrace l'origine de ce 
véritable trésor d'objets de culte vodou et makaya, reflet d'un «foisonnement rituel et mythologique».

Les recherches sur le vodou, souvent fortement empreintes de représentations et de préjugés (allant de la « satanisation », la dénégation et la pénalisation à la folklorisation ou la reconnaissance), sont réanalysées par Laënnec Hurbon sur la base d'une généalogie des statuts associés au vodou depuis la période coloniale esclavagiste, resituant ainsi le phénomène tant dans ses dimensions religieuses, économiques, culturelles, qu'identitaires, historiques et politiques. Au travers de l'étude de la littérature sociologique et anthropologique portant sur Haïti, principalement centrée sur le vodou, Kate Ramsey s'intéresse à la période de l'occupation américaine (années 1930 et 1940), et met en avant les relations entre pénalisation et "mise en scène " ethnographique des rituels, liens ayant conduit à un processus de folklorisation de cette religion. Pour répondre à la question "Quelle est l'Haïti de l'anthropologie ?», Gérarde Magloire et Kevin A. Yelvington s'attachent à une compréhension des modes de pratiques et de représentations présents au fondement du système conceptuel de l'anthropologie en Haïti, en s'appuyant sur une démarche constructiviste de mise à jour des conditions de production des discours tels qu'élaborés par trois chercheurs - Melville Herskovits, Jean Price-Mars et Roger Bastide -, divergents tant par le mode d'exposition d'écriture, la position théorique et la tradition nationale, que par la conjonction historique, le contexte social, politique et idéologique. Belle gageure, qui ne satisfera qu'à demi notre appétence d'en connaître davantage sur l'imagination anthropologique, ne dépassant qu'en partie les critiques habituelles relatives à l'ethnocentrisme, au discours colonial ou à l'effet de réification des pratiques et des représentations.

Un grand intérêt de ce dossier réside dans les divergences entre anthropologues autour de questions encore aujourd'hui débattues : la divulgation d'informations et le compte-rendu des données de terrain lors de l'écriture - l'étude effectuée par Jean Jamin à propos des écrits de Michel Leiris en est un bel exemple —, la possibilité d'une anthropologie appliquée, les influences réciproques entre chercheurs et intellectuels dans le cheminement de la pensée, la relation au politique, etc. La contribution significative d'Haïti à l'anthropologie — à travers la réhabilitation de l'apport anthropologique d'écrivains tels qu'Anténor Firmin et Jean Price-Mars — est évoquée par Carolyn Fluehr-Lobban à partir de leurs cheminements intellectuels et de leurs 
relations plus ou moins directes avec les précurseurs de cette discipline. Le texte pionnier de Firmin, De l'Égalité des races humaines (1885), constitue un des textes fondateurs de l'anthropologie générale, en tant que réponse critique aux anthropologues physiques racialistes (J. A. de Gobineau, P. Broca ou G. Le Bon), et son occultation illustre à merveille le silence de l'historiographie française sur Haïti. Rendre compte de l'histoire intellectuelle, institutionnelle et sociale du Bureau d'ethnologie de Port-au-Prince (1941) n'est pas une mince affaire, étant donné les implications politiques et religieuses qui lui sont si souvent attribuées. Rachelle Charlier-Doucet aborde ainsi les causes du rayonnement puis du déclin d'un bureau qui se voulait « le bastion de la connaissance scientifique haïtienne », cherchant notamment à former localement des ethnologues. Puis Christine Laurière nous permet une belle incursion dans le parcours d'Alfred Métraux au sein de ce qui a été un des premiers essais d'anthropologie appliquée : le projet pilote de la Vallée de Marbial.

Les divers articles de ce dossier s'enchaînent, malgré la rupture assez brutale de cette homogénéité « historique » que provoque un article consacré à l'ethnologie d'aujourd'hui. Il s'agit de la prise en compte par Chantal Collard d'Haïti comme pays «source » dans l'adoption internationale, système mis en rapport avec la circulation traditionnelle des enfants. Bien que cette étude ne soit pas dénuée d'intérêt, son intrusion nous fait regretter que le dossier ne donne qu'un bref avantgoût des recherches sur l'Haïti contemporaine. De plus, malgré la centralité du vodou dans les recherches anthropologiques, il aurait été intéressant de reconsidérer le "tournant ethnologique " de manière globale, dans ses implications notamment, même si un aperçu en est donné par Kathleen Gyssels au sujet des contes recueillis par Suzanne Comhaire-Sylvain. Densité et richesse ethnographique de données tant matérielles (objets, documents écrits, contes, nombreuses illustrations d'archives du Cidihca, du Bureau d'ethnologie, du Laboratoire d'anthropologie sociale, du Musée d'art haïtien du Collège Saint-Pierre, de la collection Lehmann, des pères du Saint-Esprit, de l'Unesco, du Musée du Quai Branly) qu'idéelles (concepts, élaboration historique d'une discipline) caractérisent toutefois ce premier numéro d'une nouvelle série fort prometteuse. 